\title{
EL FUTURO DEL MALDESARROLLO
}

\author{
José María Tortosa \\ Instituto Universitario de Desarrollo Social y Paz
}

\begin{abstract}
Resumen
El concepto de "maldesarrollo" hace referencia a una metáfora, pero a diferencia de "desarrollo", intenta referirse no a un Buen Vivir que debería buscarse sino a la constatación, primero, del fracaso del programa del "desarrollo" y, segundo, del Mal Vivir que puede observarse en el funcionamiento del sistema mundial y de sus componentes, desde los Estados nacionales a las comunidades locales. Si "desarrollo" implica un elemento normativo (lo deseable), "maldesarrollo" contiene un componente empírico (lo observable) o incluso crítico (lo indeseable).
\end{abstract}

Palabras clave: desarrollo, maldesarrollo, buen vivir.

\begin{abstract}
The concept of "maldevelopment" refers to a metaphor, but unlike the "development", this concept is not intended to refer to a "Living Well" that should be sought. It refers, first, to the failure of program of "development" and, second, to the "Bad Living" that can be observed in the functioning of the global system and its components, from nation States to local communities. If "development" implies a normative element (desirable), "maldevelopment" contains a empirical component (observable) or even critical (undesirable)
\end{abstract}

Keywords: development, maldevelopment, Living Well

La palabra desarrollo ${ }^{1}$ ha sido usada como una metáfora afortunada que comenzó a utilizarse a partir del discurso del presidente Truman de 1949. Toma pres-

1. Koldo Unceta, "Desarrollo, subdesarrollo, maldesarrollo y postdesarrollo: una mirada transdisciplinar sobre el debate y sus implicaciones", Carta Latinoamericana-Contribuciones en Desarrollo y Sociedad en América Latina, Montevideo, nº 7, 2009. 
tada de la biología la constatación de que los seres vivos se desarrollan según su código genético en un proceso natural, gradual y beneficioso. Como otras metáforas, tiene el riesgo de esconder ideología y más si consiste en indicar el objetivo a seguir, el del crecimiento, y, además, no hace ninguna referencia a los límites del mismo, como si el crecimiento fuese algo ilimitado.

La palabra maldesarrollo, por su parte, es también una metáfora. Los seres vivos sufren maldesarrollo cuando sus órganos no siguen el código, se desequilibran entre sí, se malforman. Su uso en las ciencias sociales parece haberse iniciado a partir del artículo de Sugata Dasgupta ("Peacelessness and Maldevelopment") de 1968, sigue con el libro de René Dumont y MarieFrance Mottien (Le maldéveloppment en Amérique Latine) de 1981, el de Laurence R. Alschuler (Multinationals and Maldevelopment) de 1988, aunque probablemente la obra clásica en el uso de esta palabra sea la de Samir Amin (Maldevelopment. Anatomy of a Global Failure) de 1990, sin olvidar el Insights into Maldevelopment que editó Jan Danecki en 1994 con amplia participación intercontinental. En estos dos últimos en particular, late la idea del fracaso del proyecto inicial, fracaso que se sitúa a escala mundial y no sólo a escala de los estados concretos o localidades particulares.

Sigue siendo una metáfora, pero a diferencia de "desarrollo", "maldesarrollo" intenta referirse no a un Buen Vivir que debería buscarse sino a la constatación, primero, del fracaso del programa del "desarrollo" y, segundo, del Mal Vivir que puede observarse en el funcionamiento del sistema mundial y de sus componentes, desde los Estados nacionales a las comunidades locales. Si "desarrollo" implica un elemento normativo (lo deseable), "maldesarrollo" contiene un componente empírico (lo observable) o incluso crítico (lo indeseable).

El fracaso del "desarrollo" tiene una particularidad actualmente: aunque se niegue retóricamente, sigue significando crecimiento económico medido por aumentos del Producto Interno Bruto y, sin embargo, tal vez no sea el mejor momento histórico para hablar de ello: en 2009 se ha visto reducido el beneficio de las primeras empresas en el ranking mundial que recoge la revista Forbes en su informe "Global 2000"2. De hecho, cayeron desde los 2,36 billones de dólares en 2007, a 1,63 billón en 2009. Correlativamente, la fortuna de los hiper-ricos (mil-millonarios), siempre según dicha revista ${ }^{3}$, habría disminuido en 1,4 billones de dólares en 2009 respecto a 2008.

2. Disponible en http://www.forbes.com/lists/2009/18/global-09_The-Global-2000_Rank. html.

3. http://www.forbes.com/2009/03/11/worlds-richest-people-billionaires-2009-billionaires_land.html. 


\section{QUÉ SE ENTIENDE POR MALDESARROLLO}

Tal vez sea el momento de hablar primero del diagnóstico (el maldesarrollo) antes de hablar del tratamiento o la terapia. Sin embargo, el diagnóstico es siempre con respecto a un ideal. Así sucede con la medicina clínica que compara lo observado con un determinado ideal de salud. Del mismo modo, se puede tomar como ideal el Buen Vivir para hacer un diagnóstico que, desgraciadamente, será algo más complicado que el que puede hacer un médico en su consulta: la realidad socio-económica es mucho más compleja que la biológica.

El punto de partida para dicho ideal puede ser el recurrir a las necesidades humanas básicas. La versión dominante ha sido la de reducir éstas al bienestar y tomar como satisfactores lo estrictamente monetario: el bienestar se consigue con el dinero, del mismo modo que la pobreza consiste en no disponer de un determinado montante de dólares (eso sí, a paridad de poder adquisitivo, que, de nuevo, indica la unidimensionalidad de la medida). Manfred Max-Neef y coautores reaccionaron con vehemencia ante tal reduccionismo y, reconociendo la importante distinción entre necesidad -tal vez universal-y satisfactor -más dependiente de la cultura-, proporcionaron una lista de necesidades humanas que hacía ver hasta qué punto era insuficiente la visión economicista del desarrollo, hoy todavía difundida y dominante.

Tal vez la lista de necesidades humanas de Max-Neef ${ }^{4}$ es demasiado prolija y, por tanto, ha ido al extremo opuesto de la tendencia simplista del economicismo monetarizante. Hay otras enumeraciones de necesidades humanas que pueden servir como criterios de evaluación de situaciones concretas. Johan Galtung, en el ámbito del proyecto GPID -fines, procesos e indicadores del desarrollo- de la Universidad de Naciones Unidas, propuso cuatro grandes necesidades humanas básicas, es decir, básicas porque si quedaban insatisfechas difícilmente se podía decir que la vida humana era realmente humana ${ }^{5}$.

"First things first", se puede iniciar, aunque aquí no se trate de una jerarquización de las necesidades al estilo de Maslow, por el bienestar y su mínimo exigible, la supervivencia. Pero la necesidad del bienestar no se satisface necesariamente mediante el dinero. Basta recordar las actividades económicas que quedan fuera del cálculo del PIB para ver dónde reside el problema: el autoconsumo, el trueque, el trabajo doméstico y el no asalariado en general pueden ser un satisfactor de la necesidad de bienestar sin que por ello se tenga que recurrir a una medida monetaria de dichas actividades. La seguridad es la segunda

4. Manfred Max-Neef et al., "Desarrollo a escala humana: Una opción para el futuro", Development Dialogue, No. especial, 1986, págs. 9-93.

5. VV.AA., Human needs. A contribution to the current debate, K. Lederer ed., Cambridge, Oelsgeschlager, Gunn \& Hair, 1980. 
necesidad básica a incluir en el Buen Vivir. Su contrario es la violencia que no se reduce a la violencia física sino que incluye las otras formas en las que los seres humanos consiguen de sus semejantes comportamientos o actitudes que, de no haber intervenido aquellos, no se hubieran producido. Se trata, por usar un vocabulario bien trillado, de las violencias estructurales (explotación, marginación) y de las violencias culturales que otros autores prefieren llamar violencias simbólicas y que legitiman a las anteriores. La libertad es una necesidad básica que consiste en la capacidad de decidir, libertad de y libertad para. Tiene como contrario la represión. Finalmente, la necesidad de la identidad, de la capacidad de responderse a uno mismo "quién soy yo", tiene como contrario la alienación. Son, como se ve, planteamientos muy generales, pero pueden ser útiles para definir el Buen Vivir y, consiguientemente, pueden servir para diagnosticar los casos de Mal Vivir. Pero ¿en qué ámbitos?

El desarrollo convencional ha estado oscilando entre el desarrollo nacional y el desarrollo local. Con dificultades se ha ido introduciendo el sistema mundial aunque sí han sido perceptibles las indicaciones sobre el ecosistema. De hecho, estos cuatro sistemas pueden verse como cajas chinas o como matrioshkas, una dentro de la otra pero relacionadas de forma ineludible. El sistema local forma parte del sistema nacional o estatal, que es uno de los puntos que los dedicados a los "proyectos de cooperación (al desarrollo)" suelen olvidar. Cierto que en lo local se puede intervenir con mucha más facilidad, pero eso no obsta para que lo que sucede en la escala del Estado o de la nación no acabe siendo determinante para lo local.

Pero lo mismo se puede decir sobre la escala estatal o nacional: que está inserta en el sistema mundial. Cierto, de nuevo, que el poder del Estado permite intervenciones importantes en la satisfacción (e insatisfacción) de las necesidades básicas indicadas. Pero el sistema mundial en el que se encuentra el Estado o la nación, y la crisis contemporánea es prueba de ello, se convierte en un elemento que facilita o dificulta esas intervenciones.

Para complicar el análisis, el sistema mundial se encuentra, a su vez, actuando de subsistema del ecosistema que engloba a todos los demás. De hecho, una de las razones esgrimidas para anunciar la crisis terminal del sistema mundial contemporáneo es precisamente su incapacidad para responder a los problemas de agotamiento de recursos, contaminación, calentamiento global y eventual cambio climático que no pondrían en discusión la existencia del Planeta sino de la especie humana que ha dejado esa huella ecológica indeleble y de efectos irrecuperables ${ }^{6}$.

6. James Lovelock, La venganza de Gaia, Barcelona, Planeta, 2007. Sobre la "huella ecológica" de la Humanidad sobre el Planeta: http://www.footprintnetwork.org/en/index. php/GFN/page/world_footprint/. Una cuarta parte de los "riesgos globales" enunciados 
Cruzando aquellas cuatro necesidades básicas y estos tres niveles, se obtiene el siguiente cuadro-resumen de lo que podríamos entender como maldesarrollo en el sentido de insatisfacción de necesidades humanas básicas.

Cuadro 1.- Maldesarrollo como insatisfacción de necesidades humanas básicas

\begin{tabular}{l|l|l|l}
\hline & Estatal/Local & Ecosistema & Sistema mundial \\
\hline Bienestar & $\begin{array}{l}\text { Pobreza } \\
\text { Inequidad, desigualdad } \\
\text { Estancamiento }\end{array}$ & $\begin{array}{l}\text { Calentamiento } \\
\text { Agotamiento } \\
\text { Contaminación }\end{array}$ & $\begin{array}{l}\text { Polarización } \\
\text { Periferización } \\
\text { Explotación }\end{array}$ \\
\hline Libertad & $\begin{array}{l}\text { Democracia escasa } \\
\text { Represión } \\
\text { Marginación }\end{array}$ & $\begin{array}{l}\text { Dependencia de } \\
\text { la Naturaleza, } \\
\text { sin "partenariado" }\end{array}$ & $\begin{array}{l}\text { Dependencia } \\
\text { Represión } \\
\text { Marginación }\end{array}$ \\
\hline Seguridad & $\begin{array}{l}\text { Colonización interna } \\
\text { Nacionalismo } \\
\text { Fundamentalismos }\end{array}$ & $\begin{array}{l}\text { Enajenación ante la } \\
\text { Naturaleza, pérdida } \\
\text { de raíces }\end{array}$ & $\begin{array}{l}\text { Colonialidad } \\
\text { Homogeneización } \\
\text { Reacciones } \\
\text { "identitarias" }\end{array}$ \\
\hline & $\begin{array}{l}\text { Guerra civil } \\
\text { Terrorismo }\end{array}$ & $\begin{array}{l}\text { Catástrofes de } \\
\text { origen humano }\end{array}$ & $\begin{array}{l}\text { Guerra entre } \\
\text { Estados } \\
\text { Terrorismo } \\
\text { transnacional } \\
\text { Nuclearización }\end{array}$ \\
\hline
\end{tabular}

La primera columna hace referencia a una visión del "desarrollo" algo más compleja que la que se reduce al mero crecimiento económico, sea o no acompañado por la reducción de la pobreza y, raramente, unido a la problemática de la desigualdad y la inequidad que, sin embargo, parecen tener una relación constatable con la situación tanto de los mejor situados como con la de los que están en peores condiciones ${ }^{7}$. Planteado a escala estatal, se trataría del "desarrollo nacional", pero también podría referirse al "desarrollo local". La lectura en vertical de esta primera columna indica los puntos en los que se encuentra el maldesarrollo en el mundo contemporáneo. Cierto que su incidencia será mucho mayor en los países de la periferia, después en los

en el Foro Económico Mundial, Davos 2009, se referían al tema (http://www.weforum. org/pdf/globalrisk/globalrisks09/global_risks_2009.pdf).

7. Richard Wilkinson y Kate Pickett, The spirit level. Why more equal societies almost always do better, Londres, Allen Lane, 2009; Göran Therborn, "The killing-fields of inequality", Open Democracy, 6 de abril de 2009, accesible en http://www.opendemocracy.net/article/the-killing-fields-of-inequality. 
emergentes y, finalmente, en los países centrales y en el hegemónico actual. La pobreza, la represión, el fundamentalismo o la violencia criminal no son patrimonio exclusivo de la periferia sino que se encuentra (y a veces con mayor intensidad) en los países centrales.

La segunda columna se refiere a temáticas que han estado presentes, por lo menos a nivel retórico, en algunos planteamientos del "desarrollo" como los del ecodesarrollo. Se ponen aquí para hacer ver una doble realidad: por un lado, que sus causas suelen estar más en los países centrales (y recientemente en los emergentes) que en los periféricos ya que son aquellos los mayores emisores de gases de efecto invernadero, $\mathrm{CO}_{2}$ y en general de residuos contaminantes (aunque procuren almacenarlos en países de la periferia). Por otro lado, que nos encontramos ante problemas que, aunque en algunos casos puedan ser más dramáticos en sus efectos contra las periferias en forma de catástrofes de origen humano, sin embargo, los efectos para la supervivencia de la especie y para el mantenimiento del actual sistema podrían ser generalizados.

Finalmente, la tercera columna trata de algunos aspectos del "desarrollo" que, aunque planteados por algunas escuelas (los dependentistas, los globalistas), no ha acabado de entrar en la corriente principal de las discusiones sobre el "desarrollo" (probablemente porque no había buenas terapias para dicha enfermedad) aunque sí ha entrado en los planteamientos críticos de determinados movimientos sociales como el altermundialismo. La tercera columna incluye, en efecto, las relaciones entre los diferentes actores del sistema mundial caracterizados por su asimetría en cuanto a capacidad y poder de decisión y de influencia.

Es preciso reconocer que se carece de buenos indicadores y medidas para muchos de estos componentes, incluso si se recurre a los enfoques "institucionalistas". Pero eso no significa que no sean relevantes. Incluso hay razones para preguntarse por qué se carece de ellos, no sea cosa que su ausencia sea, a su vez, un indicador más del funcionamiento "maldesarrollado" del sistema mundial contemporáneo.

Saber lo que es el Mal Vivir, incluyendo todas las necesidades básicas en los niveles reseñados (sistema local, ecosistema y sistema mundial), ayuda a definir el Buen Vivir y por dónde hay que empezar a construirlo: por los más débiles en el sistema mundial y con un contenido bien distinto al "American way of life" y a los medios utilizados normalmente para satisfacer los intereses de las élites de los países dominantes y de los sucesivos países hegemónicos. Porque no se trata de "vivir mejor" (mejor que otros, de manera indefi-

8. José Antonio Alonso y Carlos Garcimartín, Acción colectiva y desarrollo. El papel de las instituciones, Madrid, ICEI, Editorial Complutense, 2008. 
nida y no sostenible) sino que se trataría de conocer en qué consiste ese Buen Vivir, individual, colectivo y planetario, alternativo al Mal Vivir que, es importante subrayarlo, no aqueja a todo el Planeta de la misma manera.

\section{QUIÉN SE BENEFICIA DEL MALDESARROLLO}

Para tener una visión algo menos simplista de la estructura del sistema mundial hay que introducir a los países emergentes en los territorios que lo componen (y no sólo los países centrales y periféricos) y a las clases medias en los grupos sociales que actúan en el mismo y que pueden tener papeles subordinados a las otras clases o, según los casos, asumir un papel más directo, dando así una perspectiva menos alejada de la complejidad del mundo contemporáneo.

Cuadro 2.- Estructura mundial algo menos simplificada

\begin{tabular}{l|l|l|l|l}
\cline { 3 - 5 } \multicolumn{2}{c|}{} & \multicolumn{2}{l}{ PAÍS / TERRITORIO } \\
\cline { 3 - 5 } \multicolumn{2}{c|}{} & Central & Emergente & Periférico \\
\hline \multirow{4}{*}{ CLASE } & Alta & Élite imperial & Élite ascendente & Élite subordinada \\
\cline { 2 - 5 } & Media & Autocentrada & Ascendente & Ambivalente \\
\cline { 2 - 5 } & Baja & Proletariado & Parias & Hiper-marginados \\
\hline
\end{tabular}

El cuadro, además de reducir, aunque sea poco, el nivel de simplismo que siempre tienen estas herramientas más pedagógicas que descriptivas, tiene por objeto indicar quiénes se benefician del maldesarrollo contemporáneo: los que quedan por encima de la línea que, aparentemente, divide la tabla en dos mitades en su diagonal que va de "proletariado" a "élite subordinada", pero que, en realidad, deja a la gran mayoría de la población mundial como perjudicadas por el funcionamiento del sistema realmente existente. Para sólo el 7 por ciento de la población mundial (la parte más rica) se produce el 50 por ciento de los gases de efecto invernadero, al tiempo que la "huella ecológica" de los países ricos supera con creces la media mundial: para mantener su "way of life", el estadounidense medio necesitaría 9,5 hectáreas frente a las 2,7 que necesita el habitante medio del Planeta. La media en África es inferior a la unidad 9 .

9. Fred Pearce, "Consumption dwarfs population as main environmental threat", The Guardian. 16 de abril de 2009, disponible en http://www.guardian.co.uk/environment/ 2009/apr/15/consumption-versus-population-environmental-impact. 
El funcionamiento del sistema mundial contemporáneo es "maldesarrollador" porque es un sistema basado en la eficiencia que trata de maximizar los resultados, reducir costes y conseguir la acumulación incesante de capital. Ésa es la regla de juego que para nada es atemperada por la "mano invisible" de los sentimientos morales de que hablaba Adam Smith, es decir, por el sentido de la responsabilidad. Si "todo vale", el problema son las mismas reglas del juego dictadas y cambiadas desde arriba para satisfacer aquellos intereses. En otras palabras, el sistema mundial está maldesarrollado por su propia lógica y es a esa lógica a donde hay que dirigir la atención ${ }^{10}$. Este maldesarrollo así descrito está producido por la interacción simultánea de cuatro conflictos que generan cuatro violencias, que no son tanto de "los de abajo" contra "los de arriba", que suele ser poco frecuente, sino que son la más cotidiana y frecuente lucha de "los de arriba" contra "los de abajo". El resultado de esas luchas (violencia estructural acompañada a veces de violencia directa) es el orden social realmente existente.

1. En primer lugar, hay una lucha de clases, más allá de los Estados, de lo que se podrían llamar las élites a escala mundial, cosmopolitas, o la también llamada cosmocracia ${ }^{11}$, contra el resto de la población del Planeta, ésta sí dividida en Estados y naciones y con tendencia al nacionalismo. Se trata del grupo dominante a escala mundial, transnacional, relativamente bien organizado y con evidente conciencia de sus intereses y del modo de defenderlos, aunque su poder no es cierto que sea total: hay, evidentemente, conflictos internos y hay límites a la acción posible. Son, de alguna forma, los productores principales y sustentadores del maldesarrollo contemporáneo. Casi se puede decir que esta cosmocracia vive en otro Planeta, en cualquier caso en otro mundo de bienestar, identidad, libertad y seguridad, con muy escaso contacto con el resto de sus habitantes, pero enzarzada en una lucha de clases contra todos los demás con tal de mantener el orden de sus privilegios.

2. En segundo lugar, introduciendo los Estados, hay una lucha de los países centrales contra los países periféricos. La lógica de la cosmocracia es la de utilizar al Estado en función de sus propios intereses y, desde ese punto de vista, nada más lejos de la realidad que la supuesta "obsolescencia" de los Estados dentro del proceso de "globalización" definido más en términos ideológicos que empíricos: los países centrales han visto reforzadas sus estructu-

10. Varios autores., Flat world, big gaps. Economic liberalization, globalization, poverty and inequality, K.J. Jomo y J. Baudot eds., Zed Books, Londres, 2007.

11. Martín Ortega Carcelén, Cosmocracia. Política global para el siglo XXI, Síntesis, Colección Ciencias Políticas, Madrid, 2006; Jeff Faux, The global class war, John Wiler \& Sons, Hoboken, NJ, 2006. 
ras estatales, también en la Unión Europea, con ejércitos, policía, legislación más o menos represiva, vigilancia más o menos legal, violación de la intimidad, control de aduanas, control de fronteras en particular para el caso de los inmigrantes, defensa diplomática de "sus" empresas y demás. Al mismo tiempo, aconsejaban a los países periféricos el desmantelamiento del Estado o su conversión en "estado mínimo". La periferización causada inicialmente por la colonización, es decir, por la incorporación forzosa de los territorios periféricos al funcionamiento del sistema en función de los intereses de las élites de los países centrales, fue mantenida durante la descolonización y la aparición del neocolonialismo. En este contexto se sitúan los discursos sobre el "desarrollo" como una legitimación más de esta estructura centro-periferia ${ }^{12}$. Posteriormente, se ha reforzado todavía más en la etapa de exaltación de la ideología neoliberal (poco desarrollista) que los países centrales no han practicado con tanto entusiasmo como han impuesto a los países periféricos ${ }^{13}$. Recientemente, ha llegado a su cenit en la etapa neoconservadora en la que se ha usado la "seguridad" como mecanismo para asegurarse la victoria en esta lucha con los consiguientes efectos sobre la desigualdad y la pobreza. El resultado es el orden del maldesarrollo y queda por ver qué efectos tendrá sobre estas cuestiones el retorno del Estado después de la caída del neoliberalismo ${ }^{14}$.

3. Después está la lucha de clases dentro del Estado, mucho más aguda en los países periféricos, y atenuada en los centrales gracias al reparto del expolio de los países periféricos por parte de los países centrales. De haber lucha de clases "desde abajo" es más en los países periféricos que en los centrales. En estos últimos hay un acuerdo tácito, que incluye a las llamadas fuerzas de izquierdas (partidos y sindicatos), para suavizar el conflicto mediante una mayor explotación de los países periféricos ${ }^{15}$. Así, por ejemplo, en 2006, el $24 \%$ de los resultados de las empresas españolas del Ibex, principal índice de referencia de la Bolsa española, provenía de América Latina, con especial incidencia en las grandes (55\% en el BBVA; 45\% en Repsol YPF; 37\% en el Banco

12. Gilbert Rist, El desarrollo: historia de una creencia occidental, Madrid, IUDC-La Catarata, 2002.

13. Para una enumeración de los contenidos del "consenso de Washington", véase Alberto Acosta, Desarrollo glocal, Corporación Editora Nacional, Quito, 2005, pp. 18-19.

14. Immanuel Wallerstein, "2008: The demise of neoliberal globalization", Commentary $\mathrm{N}^{\circ} 226,1^{\circ}$ de febrero de 2008, disponible en http://www.binghamton.edu/fbc/ 226en.htm.

15. Eso explica las perplejidades de la "izquierda" en el país hegemónico y también en los países centrales como la Unión Europea. Para este último caso, véase Hisham Bustani, "Pensieri fuori stagione. Per una critica della sinistra europea", Senza Censura, XIV, 28 (2009) 4-6, disponible en http://www.senzacensura.org/public/pdf/SC_28_09_it.pdf, traducido y actualizado en http://www.rebelion.org/noticia.php?id=83413 
Santander; $33 \%$ en Telefónica y $30 \%$ en Endesa $)^{16}$. Lo que es común a países centrales y periféricos es la lucha de clases "desde arriba" para mantener situaciones de inequidad interna y externa con un mínimo de violencia directa. Orden, al fin y al cabo.

4. Finalmente, está la hegemonía contemporánea de los Estados Unidos, lucha del país (o, mejor, de sus élites) contra el resto para imponer su "way of life", aunque con aliados, y cuyos efectos algunos autores han puesto de manifiesto. George Soros ${ }^{17}$ está convencido de que "el mayor obstáculo para un orden mundial estable y justo son los Estados Unidos". La hegemonía de los Estados Unidos es crucial para entender algunos problemas actuales y esto en varios sentidos. Ante todo, porque la agenda de sus élites se impone al mundo. Al fin y al cabo, lo que llamamos hegemonía no es otra cosa que la capacidad de imponer la satisfacción de los intereses de las propias élites al conjunto del sistema utilizando un mínimo de violencia. No son, pues, ajenos a sus efectos en el maldesarrollo. Pero también es crucial porque la historia del sistema mundial, por lo menos los últimos 500 años, es la lucha entre los países centrales por lograr esa hegemonía, generando así un conflicto estable de rivalidad que se ha ido resolviendo sucesivamente mediante el recurso a la violencia, que es el caso de las llamadas "guerras mundiales", es decir, guerras por la hegemonía entre países centrales ${ }^{18}$. Finalmente, es crucial porque, desde diversos ángulos, se está afirmando la decadencia de la hegemonía estadounidense aunque no hay acuerdo sobre qué tipo de mundo sustituiría al "siglo americano". El tiempo dirá, pero mientras tanto el maldesarrollo del orden internacional continúa. ¿Es sostenible esa situación?

\section{CAMBIO DE ÉPOCA}

Xavier Albó publicó en 1986 un artículo ${ }^{19}$ en el que planteaba lo que sucedería cuando los pueblos originarios despertasen en Bolivia y, se puede extender, en América Latina. En buena medida ha sucedido o está sucediendo. Los respectivos artículos de las constituciones boliviana y ecuatoriana son prue-

16. El País (Madrid), 18 de noviembre de 2007.

17. George Soros, La burbuja de la supremacía norteamericana: Cómo corregir el mal uso del poder de los Estados Unidos, Debate, Barcelona, 2004.

18. Luciano Vasapollo, James Petras y Mauro Casadio, Potencias en conflicto. La pugna por la hegemonía mundial, Viejo Topo, Barcelona, 2007; Varios autores, Crisis de hegemonía de Estados Unidos, M.A. Gandásegui hijo, coord., Siglo XXI y CLACSO, México, 2007.

19. Xavier Albó, "Cuando el gigante despierte", Cuarto Intermedio (Cochabamba), 1 (1986) 3-26. Reproducido como "El gigante despierta", íbidem, 77 (2005) con ocasión del ascenso de Evo Morales. 
ba de ello: en 1986 a poca gente se le habría ocurrido introducir "Sumak Kawsay" o "Suma Qamaña" o "Qhapaj Ñan" en un texto constitucional tradicionalmente reservado a los criollos. El detalle forma parte de la aparición de nuevos actores en la escena local que se convierten en actores en la escena internacional desequilibrando todavía más un sistema tan alejado del equilibrio como para que estos "despertares" puedan tener un efecto mucho mayor del que tendrían de estar el sistema en equilibrio.

Pero no sólo se trata de la emergencia de los pueblos indígenas como actores locales-globales. También es cuestión de la incorporación de nuevos actores globales en términos de países, puesta de manifiesto por la entrada de los países "emergentes" en la escena mundial y de sus élites en las listas de mil-millonarios o asistentes a encuentros "globales". Hay algunos elementos incipientes en el sistema mundial que, en paralelo con lo ya indicado, no está claro si van a seguir o no pero que, de seguir, van a alterar de forma profunda el funcionamiento del mismo. Se trata de los cambios en la composición de la élite mundial (su occidentalización se va reduciendo) y los cambios en las relaciones Norte-Sur (o, si se prefiere, países centrales-países periféricos).

Los escenarios publicados por la CIA a finales de 2008 con el horizonte de 2025 son sintomáticos ${ }^{20}$. Manteniendo el formato cuatripartita de los ejercicios anteriores, pero incrementando su etnocentrismo, los escenarios serían:

1. Un mundo sin Occidente en el que las nuevas potencias suplantan a Occidente como líderes de la escena mundial.

2. Sorpresa de Octubre en el que se produce el impacto de la falta de atención al cambio climático ampliando las opciones para el mundo bajo forma de impactos inesperados

3. Los BRIC se queman, es decir, disputas sobre las potencias mayores sobre recursos vitales, en particular entre la China y la India (Brasil y Rusia son los otros dos componentes del grupo BRIC)

4. La política no es siempre local en el que redes no-gubernamentales emergen para establecer una agenda internacional sobre el medio ambiente con lo que eclipsan a los gobiernos.

En general, un mundo en el que los Estados Unidos pierden puestos de forma perceptible $^{21}$. Y, probablemente, de forma mayor y más acelerada de lo que los expertos de la CIA están dispuestos a reconocer y que las sucesivas cumbres latinoamericanas sin los Estados Unidos se encargan de escenificar.

20. Global trends 2025: A transformed world, noviembre de 2008, accesible en http://www.dni.gov/nic/PDF_2025/2025_Global_Trends_Final_Report.pdf . Puede verse el anterior (Mapping the global future 2020) en http://www.foia.cia.gov/2020/2020.pdf

21. Johan Galtung (The fall of the US empire - and then what? Successors, Regionalization or Globalization? US Fascism or US Blossoming?, Transcend, próximo) encuentra 15 contradicciones que llevarían a la caída de dicho imperio. 
Todo parece indicar que la crisis económica contemporánea puede traer consigo una mayor alteración de los componentes de la élite y una aceleración de los elementos "reformistas" y, en cualquier caso, menos neoliberales con respecto a los observados en los años anteriores. Además, para enfrentarse a esta crisis, ya es obvio que no puede hacerse pensando sólo en la economía sino que hay que introducir otras variables ${ }^{22}$.

Tal vez el punto en el que mejor pueden observarse los cambios producidos en las relaciones entre países centrales y periféricos sea el petróleo. El petróleo se ha convertido en un arma en manos de los gobiernos del Sur. Problemática ${ }^{23}$, pero real. Y mucho más si se lo ve acompañado de los "sovereign wealth funds" $\left(\mathrm{SWF}^{24}\right)$, cuentas de inversión controladas por gobiernos (Abu Dhabi Investment Authority, Kuwait Investmente Authority y así sucesivamente) con las que invierten en el exterior para asegurarse fuentes de ingresos que no dependan del petróleo. Es, pues, su forma de prepararse para una "economía postpetrolera", sus fondos se calculan en billones de dólares y sus compras o rescates comienzan a ser notorias en particular de fuentes financieras anglosajonas.

Al mismo tiempo, la presencia de la China en los escenarios internacionales ha introducido cambios sustanciales en las alianzas y competencias entre superpotencias. La presencia de la China, por ejemplo en África, está reduciendo la de los Estados Unidos. Aunque la distancia de renta entre países, en su conjunto, esté aumentando a escala mundial, la capacidad de negociación de muchos países del Sur frente a los del Norte se ha incrementado como han crecido las posibilidades y las actuaciones en términos infrecuentes hace relativamente poco tiempo, con una mayor intervención de los gobiernos, más frecuentes nacionalizaciones y menores aceptaciones de los dictados de las instituciones del Norte no hace mucho obedecidas sin mayores problemas. Lo cual no quiere decir que no haya intentos de reducir o incluso suprimir algunas de estas rebeldías tanto las que se producen frente a los países centrales como las que se dan frente a las respectivas élites locales.

22. David Korten, "Beyond the bailout: Agenda for a new economy", Yes!, invierno de 2009, accesible en http://www.yesmagazine.org/article.asp?ID=3050\&utm_source= feb09\&utm_medium=email\&utm_campaign=N3_topArticl.

23. Kurt Zenz House, "OPEC and the Prisoner's Dilemma", Bulletin of the Atomic Scientists Newsletter, 17 de diciembre de 2008, accesible en http://thebulletin.org/webedition/columnists/kurt-zenz-house/opec-and-the-prisoners-dilemma. En todo caso, no parece que sea igualmente problemática para países con poca población como las monarquías del Golfo Pérsico que para países como Venezuela, Irán o Rusia. Véase Jean-Michel Bezat, «Inquiétude au pays de l'or noir», Le Monde, 17 de diciembre de 2008.

24. Véase "Sovereign Wealth Funds", Times Topics, The New York Times http://topics.nytimes.com/top/reference/timestopics/subjects/s/sovereign_wealth_funds/index.html 
Tal vez ese sistema mundial esté acabando y precisamente la caída de los Estados Unidos acelere el fin del sistema ${ }^{25}$. Sin embargo, los cambios en el sistema mundial no se reducen a los cambios en las distintas formas de detentar el poder dentro del mismo, desde la clase social a la hegemonía pasando por la estructura de poder centro-periferia, con los distintos "gigantes" que se despiertan. Con independencia de la tantas veces anunciada crisis terminal del sistema, y de la que sigue habiendo argumentos para dudar, el hecho es que en la presente coyuntura se acumulan y retroalimentan diferentes crisis con efectos difícilmente previsibles.

1. Está, en primer lugar, la crisis económica global ${ }^{26}$ ya citada. El Global Risks 2008 que se presentó en Davos en febrero de 2008 tenía motivos sobrados para preguntase si se iba a entender esta crisis y si se iba a saber mitigar ${ }^{27}$. De momento las respuestas son negativas y, no sin cierta impotencia, Dennis C. Blair, Director de Inteligencia Nacional, comenzaba su informe anual ante el Comité de Inteligencia del Senado sobre las amenazas previsibles diciendo que "a corto plazo, la principal preocupación relativa a la seguridad de los Estados Unidos es la crisis económica global y sus implicaciones geopolíticas" 28 , añadiendo la falta de consenso sobre causas y remedios. "A pesar de que ya lleva un año entre nosotros,", dice, "los economistas discuten si y cuándo podría tocar fondo. Algunos incluso temen que la recesión se haga todavía más profunda y alcance el nivel de la Gran Depresión".

2. La fuente de acumulación de beneficio que fue el sector financiero ya no lo es. En los últimos años, era preferible especular mediante productos financieros sofisticados y herméticos que producir si lo que se quería era una abundante acumulación de capital. Ahora, con el punto anterior, esto ya no

25. Jorge Beinstein, J., "Entre la recesión y el colapso. El hundimiento del centro del mundo", ALAI, América Latina en movimiento, 6 de mayo de 2008. Disponible en http://alainet.org/active/23919. También Ian Bremmer y Flynt Leverett, "The age of disorganization", Foreign Policy, abril 2009, accesible en http://www.foreignpolicy. com/story/cms.php?story_id=4894. Igualmente Minqi Li, The rise of China and the demise of the capitalist world economy, Nueva York, Monthly Review Press, 2009. Dilip Hiro, After empire: The rise of a multipolar world, Nueva York, Nation Book, 2009.

26. Varios autores. La primera crisis global del siglo XXI: Miradas y reflexiones, E. Gudynas comp., Montevideo, CLAES, 2009, disponible en http://www.iudesp.ua.es/documentos/ClasesCrisisGlobal.pdf; Juan Torres López con Alberto Garzón Espinosa, La crisis financiera. Guía para entenderla y explicarla, Attac España, 2009, disponible en http: //hl33.dinaserver.com/hosting/juantorreslopez.com/jtl/images/stories/libro_attac.pdf.;

27. http://www.weforum.org/pdf/globalrisk/report2008.pdf. En castellano en http://www. weforum.org/pdf/globalrisk/GlobalSpanish.pdf

28. Dennis C. Blair, "Annual Threat Assessment of the Intelligence Community for the Senate Select Committee on Intelligence", 12 de febrero de 2009, disponible en http:// intelligence.senate.gov/090212/blair.pdf. 
funciona y todavía no se ve de dónde vendrá el grueso del beneficio futuro si es que hay alguno. La llamada "sociedad del conocimiento" podría repetir lo sucedido con la "Nueva Economía" o la "burbuja.com".

3. Desequilibrios globales producidos por los desequilibrios estadounidenses. Su déficit (federal, comercial) y sus deudas (públicas, familiares, empresariales) son insostenibles y, con ello, la moneda de referencia mundial, el dólar, puede entrar en barrena en cualquier momento sin que esté clara la alternativa ${ }^{29}$. El euro o el yen no tienen la fuerza (política, no sólo económica) necesaria para sustituir al dólar, pero son perceptibles las propuestas alternativas que van desde la moneda común de los 6 países del Consejo de Cooperación del Golfo al "sucre" latinoamericano pasando por la ACU, unidad de cuenta asiática, semejante a lo que fue el ECU, propuesta en ASEAN, Asociación de Países del Sureste Asiático. Tenemos, pues, una situación de "ya no" pero, al mismo tiempo, de "todavía no". El dólar ya no cuenta tanto pero todavía no hay una alternativa viable.

4. La crisis alimentaria global. Es una forma de decir que el hambre se está disparando en el mundo. La ha producido una mezcla de aumento de demanda por parte de los ricos para propósitos también no alimentarios (energéticos), una reducción de la producción en las zonas hambrientas por cuestiones ambientales y una disminución de la capacidad de compra en importantes sectores de la población mundial ${ }^{30}$. Para los cínicos, eso puede ser un problema a olvidar ya que sólo afectaría a los hambrientos (más de 900 millones en 2009 según la $\mathrm{FAO}^{31}$ ) o a los que mueren de hambre. Pero igual que se habló del "boomerang de la deuda" que, lanzada por los países enriquecidos contra los empobrecidos empobreciéndolos más, se volvía contra los que la habían lanzado en términos de inestabilidad financiera y crisis de sobreproducción, se puede hablar ahora del "boomerang del hambre": inestabilidad política y militar, nuevas enfermedades o el aumento de oleadas migratorias son razones para que los países enriquecidos tuviesen que practicar alguna forma de "egoísmo ilustrado", es decir, saber que resolver este proble-

29. Alfredo Jalife-Rahme, "LEAP/Europe 2020 vaticina inminente quiebra del dólar", La Jornada (México), 22 de abril de 2009.

30. Robert Pearlberg, "The real hunger crisis", Foreign Policy, 8 de abril de 2009, accesible en http://experts.foreignpolicy.com/posts/2009/04/08/the_real_hunger_crisis.

31. FAO, El estado de la inseguridad alimentaria en el mundo 2008, Roma, Organización de las Naciones Unidas para la Agricultura y la Alimentación, 2008, accesible en ftp:// ftp.fao.org/docrep/fao/011/i0291s/i0291s00.pdf. A principios de 2009 el cálculo llegaba a 1.000 millones de personas infra-alimentadas (Financial Times, 29 de marzo de 2009, disponible en http://www.ft.com/cms/s/0/252ea7b8-1a2f-11de-9f91-0000779fd 2ac.html) 
ma es en interés propio. No parece que lo vayan a hacer ${ }^{32}$. Una vez más, mezcla de "teoría del gorrón" y "dilema del prisionero" y la lógica de un sistema que busca el beneficio por encima de cualquier otra consideración ${ }^{33}$.

5. Crisis energética. Tiene que ver con el hambre (los biocombustibles) pero, básicamente, significa que ya hemos llegado o estamos a punto de llegar al "pico del petróleo", ese momento en el que el aumento del consumo se hace a costa de la reducción de reservas. El descubrimiento de nuevos yacimientos, como el del Brasil, no altera sensiblemente el problema general aunque sí el valor de las acciones de las correspondientes petroleras privadas (las estatales van por otro lado) y es que no hay modo de responder a las crecientes demandas de los nuevos países industrializados (la China, la India, es decir, una parte muy importante de la población mundial) y, por más que se esté trabajando en alternativas eólicas, fotovoltaicas o incluso nucleares, todavía no hay modo de encontrar algo que sustituya a la energía tal y como la conocemos y que van de los coches a los plásticos. De ahí las complicadas contradicciones entre el reconocimiento de los "derechos de la Naturaleza", como se ha venido reivindicando, y las presiones para la extracción de este "oro negro" que fluye por las venas del sistema dándole la "vida". Al sistema, no a la Naturaleza. El optimismo tecnológico pudo contrarrestar el pesimismo inicial de "los límites del crecimiento", pero no siempre la tecnología tiene que ser omnipotente.

6. Crisis ambiental. Si se puede dudar del cambio climático (difícil de vaticinar) o del calentamiento global (constatable aunque no se sepa por cuánto tiempo), es preciso reconocer que, por lo menos coyunturalmente, el Planeta está sometido a tensiones medioambientales severas que afectan al hambre, pero no sólo: también a la falta de agua en ciudades por agotamiento de nieves perpetuas, deshielo en el Ártico y de glaciares, sequías, lluvias torrenciales, ciclones, tsunamis, temperaturas inusuales, especies en extinción, agotamiento de caladeros para la pesca, cambios en la duración de El Niño (ENSO) y La Niña...

32. Véase Sarah Anderson, John Cavanagh y Janet Redman, "Skewed priorities", Institute for Policy Studies, 24 noviembre de 2008, disponible en http://www.ips-dc.org/ reports/\#912, comparando los más de 4 billones de dólares comprometidos en 2008 por los Estados Unidos y la Unión Europea para los diversos rescates bancarios con los 90.000 millones que han dedicado a la ayuda al desarrollo en 2007 y que es previsible que hayan disminuido en 2008.

33. Mientras el Banco Mundial preveía cien millones de personas padeciendo hambre severa y la FAO afirmaba que había 37 países con una urgente necesidad de alimentos, se reconocían los incrementos espectaculares en los beneficios de las empresas con intereses en la alimentación como Monsanto, Cargills o Mosaic Company (Geoffrey Lean, "Multinationals make billions in profit out of growing global food crisis", The Independent (Reino Unido), 4 de mayo de 2008). 
Algunos estudios patrocinados por el gobierno de los Estados Unidos ${ }^{34}$ subrayan la novedad de las violencias que se derivan de la combinación de estos factores. Según dicho estudio, las Academias Militares tendrán que revisar sus programas convencionales ante el carácter poco convencional de las nuevas amenazas a la seguridad. Se puede añadir que no sólo las Academias Militares, sino también la Academia tout court tendría que revisar muchas de sus teorías.

$\mathrm{Si}$, a corto plazo, no se ven "clases peligrosas" para las élites mundiales y los desafíos del Sur son gestionables sin excluir la intervención militar, y mientras se "condensan" las crisis recién indicadas, parecería que el mayor cambio sería en torno al cambio de hegemonía en el sistema que, a su vez, podría introducir cambios de mayor magnitud en un sistema tan alejado del equilibrio como el que se acaba de describir. Unidas a las debilidades que acompañan al "American way of life", está el error cometido por los neoconservadores que han gobernado con George W. Bush pretendiendo alcanzar un "imperio" (basado en el "poder duro") en circunstancias que, objetivamente, no lo permitían y que tal vez sólo daban para una "hegemonía" 35 . Si el presidente Barack Obama va a conseguir revertir la tendencia decadente, está por ver $^{36}$. De momento, la acumulación de pequeñas derrotas, pero numerosas, hacen pensar en el tormento chino de "los mil cortes": pequeñas heridas, ninguna de las cuales, separadamente, consigue acabar con la persona pero que, juntas, consiguen su propósito. Immanuel Wallerstein ${ }^{37}$ aplica la metáfora a las sucesivas pequeñas derrotas de los Estados Unidos en América Latina a cuya lista habría que añadir las producidas después de 2005. Pero eso no significa que tenga que seguir así por necesidad, aunque no sería la primera vez que una potencia hegemónica tiene sus propios auges y caídas, por lo menos según algunos cómputos. Fue el caso de la Gran Bretaña tal y como lo planteaba George Modelski ${ }^{38}$.

34. Nathan P. Freier, "Known unknowns: Unconventional "strategic shocks" in defense strategy development", PKSOI Papers, Strategic Studies Institute, 4 de noviembre de 2008, accesible en http://www.strategicstudiesinstitute.army.mil/pubs/display.cfm?pub $\mathrm{ID}=890$

35. Para la lucha política interna entre los partidarios de una "globalización empresarial" (hegemonía) y una "globalización imperial" (imperio), Mark Engler, How to rule the world, Nueva York, Nation Books, 2008.

36. Alfredo Toro Hardy, “¿Podrá Estados Unidos recuperar su hegemonía?”, Rebelión, 11 de abril de 2009, disponible en http://www.rebelion.org/noticia.php?id=83600

37. Immanuel Wallerstein, "Death by a thousand cuts", Commentary $\mathrm{n}^{\circ} 160,1^{\circ}$ de mayo de 2005. Disponible en http://www.binghamton.edu/fbc/160en.htm

38. George Modelski, Long cycles in world politics, Seattle, University of Washington Press, 1987. 
Immanuel Wallerstein, recordando a Andre Gunder Frank ${ }^{39}$, ha planteado los tres horizontes en los que se puede pensar el futuro. El primero es el corto plazo y ahí, dice, por lo general sólo nos queda la opción del mal menor según la perspectiva de cada cual. A medio plazo, en cambio, se sitúan opciones más importantes. Él las ejemplifica entre Davos y Porto Alegre en esta crisis que él afirma se trata de una crisis sistémica: entre el Foro Económico Mundial (la lógica del beneficio) y el Foro Social Mundial (la lógica de la solidaridad). Aquí serían entre el "American way of life" y el "Sumak Kawsay", "Suma Qamaña" o Buen Vivir. Finalmente, hay opciones que tienen como horizonte el largo plazo, y se trata de la toma de posición y búsqueda de aliados de cara a un mundo que puede ser menos jerárquico y desigualitario que el presente, o mucho más. El futuro del maldesarrollo no depende de leyes históricas, universales e inalterables sino de las decisiones que tomen los actores sociales desde la escala local a la global. El Buen Vivir, como proyecto, puede ser una alternativa relevante

39. Immanuel Wallerstein, "RememberingMonthly Review. 